\title{
Fringe Pattern of an Oscillating Fabry-Perot Interferometer
}

\author{
K. D. Mielenz
}

(December 17, 1963)

\begin{abstract}
The dependence of the fringe pattern of an oscillating Fabry-Perot interferometer upon the vibration amplitude is discussed experimentally and theoretically. If the fringes of the stationary interferometer are wide, the fringe pattern tends to disappear at certain values of the amplitude. If the stationary fringes are narrow, a splitting of fringes occurs at certain amplitudes. In both cases, the stationary pattern reappears, with reduced contrast, at intermediate amplitudes.

The practicability of using these effects for the measurement of vibration amplitudes is discussed.
\end{abstract}

\section{Introduction}

In a recent paper [1], ${ }^{1}$ a photoelectric spectrum analyzer was described that consisted of a Fabry-Perot etalon with a piezoelectric ceramic tube incorporated into the spacer. If an a-c voltage of circular frequency $\omega$ is applied to the ceramic, the length of the etalon, $d$, varies with time, $t$, according to

$$
d=d_{0}+d_{1} \cos \omega t
$$

The static length of the etalon, $d_{0}$, was $10 \mathrm{~cm}$ in one experiment, $8 \mathrm{~mm}$ in another. The vibration amplitude, $d_{1}$, is of the order of a few wavelengths. It is proportional to the peak voltage applied, $V_{1}$; the ratio was, in both cases, $d_{1} / V_{1}=1.5 \mathrm{~m} \mu / \mathrm{v}$. A 60 -cycle a-c voltage was used. For both experiments, the green line of $\mathrm{Hg} 198$ was chosen to illuminate the interferometer.

The visual observation of the Haidinger fringe patterns of these oscillating interferometers reveals a peculiar behavior:

As the vibration amplitude increases, the fringe pattern of the 10 -cm etalon almost disappears at certain discrete values of the amplitude and reappears at intermediate values; figure 1.

With the S-mm etalon, on the other hand, the pattern also reappears at certain amplitudes but, instead of vanishing at intermediate amplitudes, it appears as a double pattern; i.e., with each fringe split into two, figure 2 .

The contrast of the pattern in both cases gradually decreases as the amplitude increases.

Fringe disappearance, as in the first case, is well known from oscillating two-beam interferometers. Described by Osterberg more than 30 years ago [2], it is now the basis of an established technique of measuring small vibration amplitudes [3].

As of this writing, the obviously more complex behavior of the oscillating multiple-beam Fabry-Perot interferometer, however, has not yet been explained.

\section{Theory}

Consider an etalon of length $d$, and let $T$ and $R$ be the transmission and reflection coefficients of the etalon plates. Its transmission is

with

$$
A(\nu, d, i)=T^{2}\left(1+R^{2}-2 R \cos \varphi\right)^{-1},
$$

$$
\begin{aligned}
& \varphi=\beta \nu, \\
& \beta=(4 \pi / c) d \cos i, \\
& c=\text { speed of light, } \\
& \nu=\text { frequency, } \\
& i=\text { angle of observation. }
\end{aligned}
$$

\footnotetext{
${ }_{1}^{1}$ Figures in brackets indicate the literature references at the end of this paper.
} 


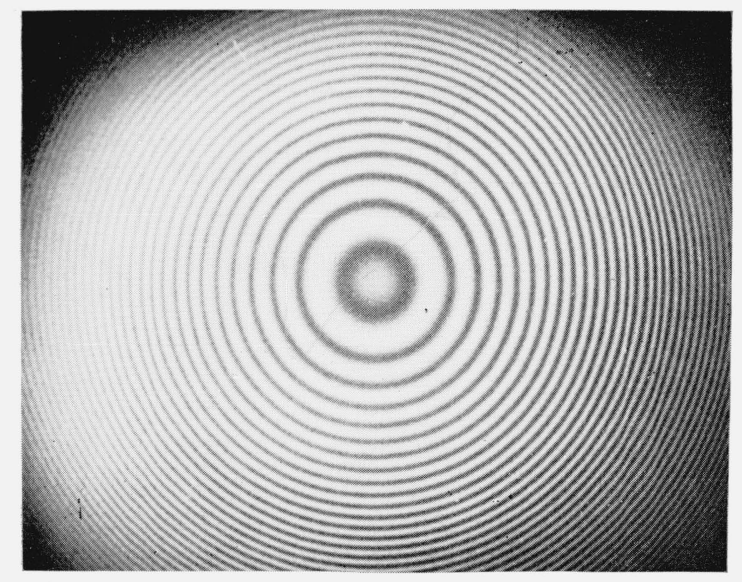

a

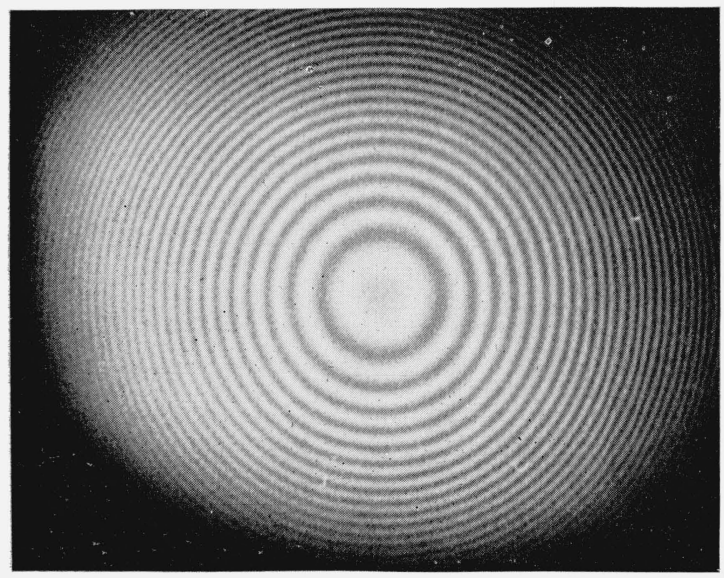

C

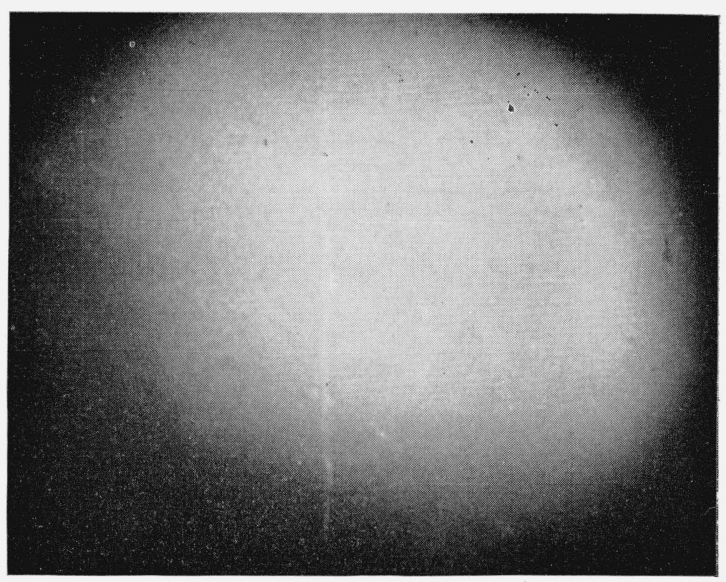

b

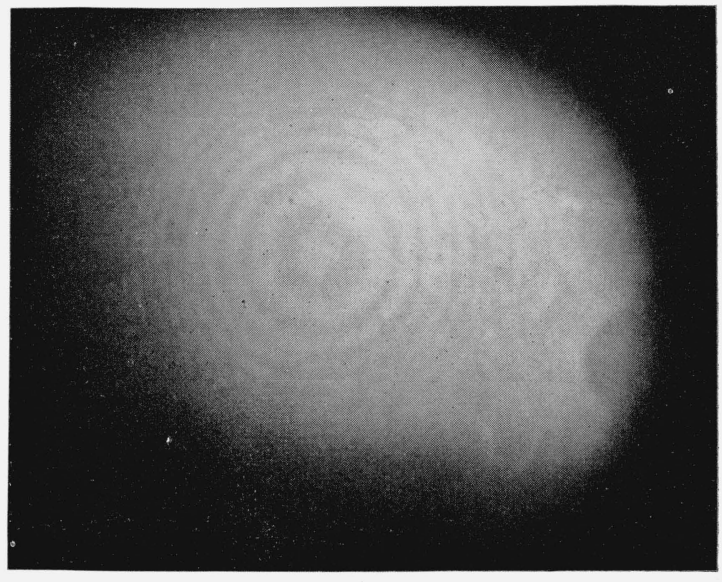

d

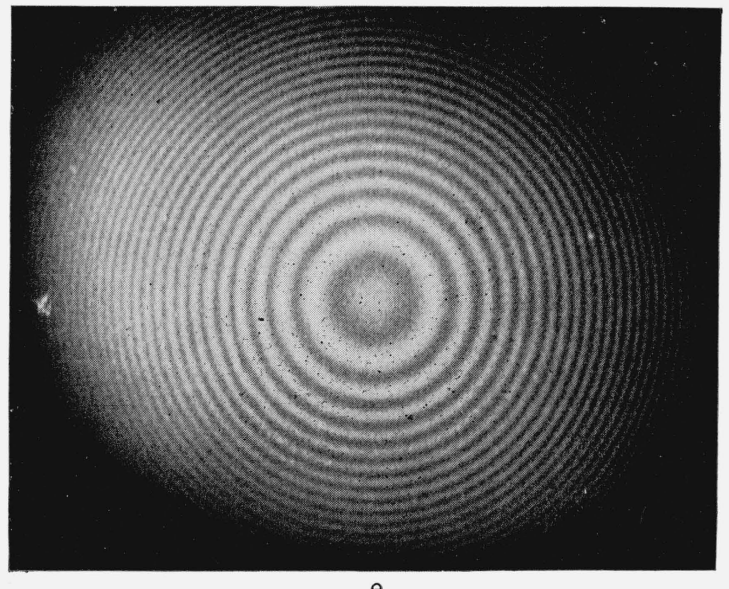

e

Figure 1. Fringe pattern of oscillating 10-cm etalon, for $\mathrm{V}_{1}=0,68,110,154$, and $201 v$. 


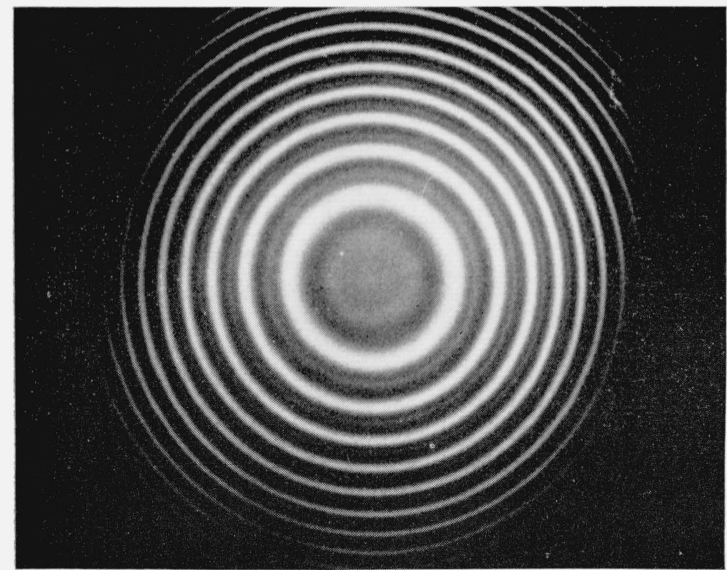

a

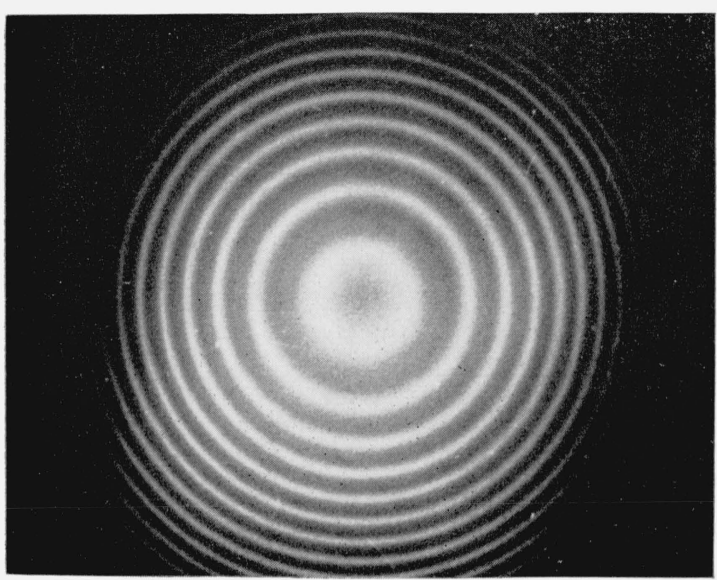

C

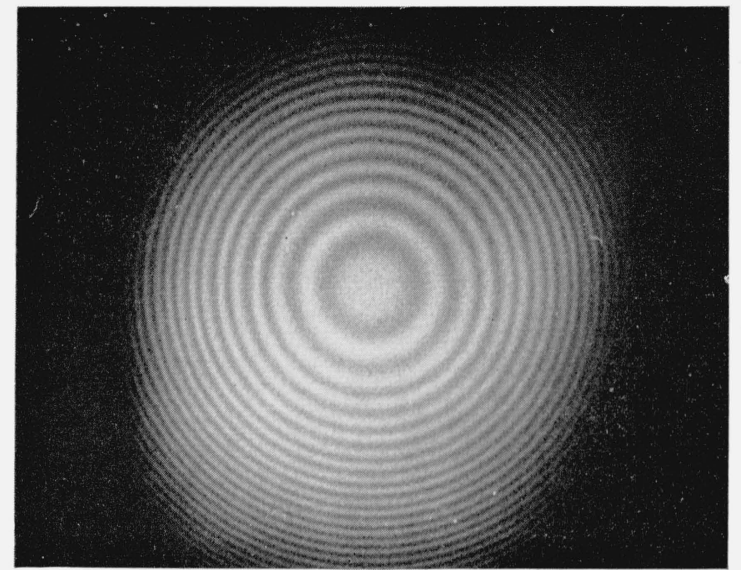

b

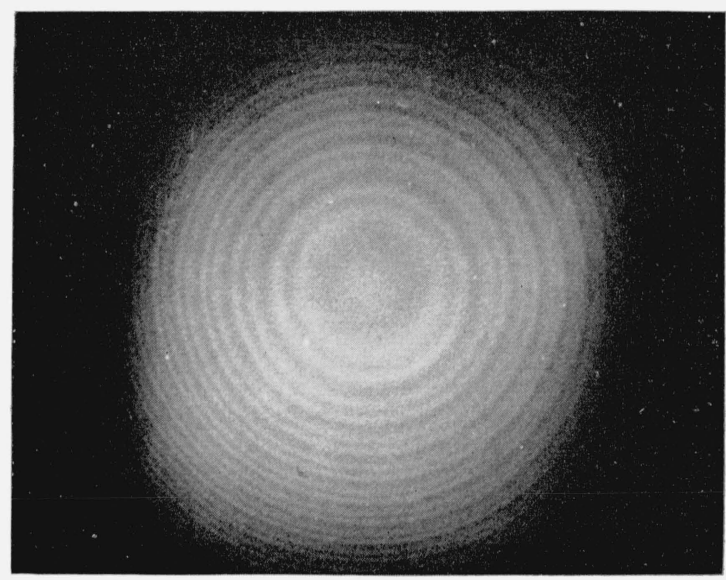

d

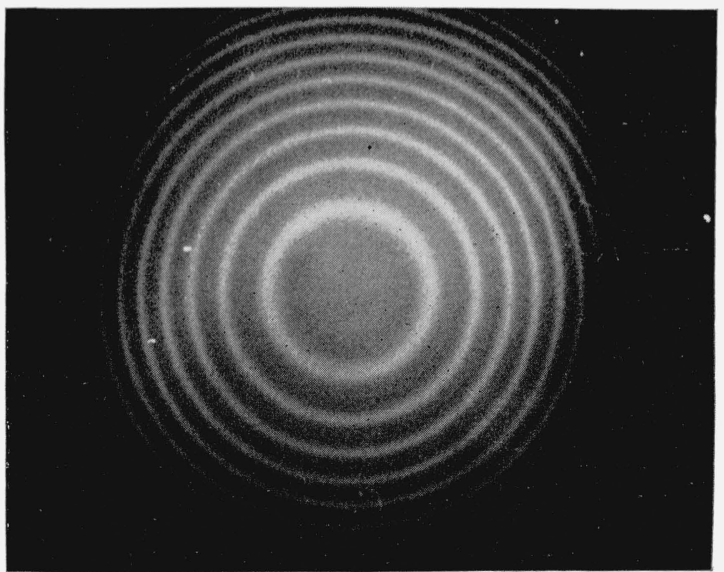

e

Figure 2. Fringe pattern of oscillating 8-mm etalon, for $\mathrm{V}_{1}=0,46,96,148$, and $178 v$. 
Let

$$
D(\nu)=C e^{-\alpha^{2}\left(\nu-\nu_{0}\right)^{2}}=C e^{-(\alpha / \beta)^{2}(\varphi-\Phi)^{2}}
$$

be the Doppler distribution of the illuminating light, $\nu_{0}$ being its principal frequency. Here,

$$
\begin{aligned}
C, \alpha & =\text { constants }, \\
\phi & =\beta \nu_{0} .
\end{aligned}
$$

Monochromatic light, of frequency $\nu$, is then observed in a given direction $i$ with the intensity

$$
I_{\nu}(d, i)=D(\nu) A(\nu, d, i) .
$$

The total intensity observed in that direction is

$$
I(d, i)=\int_{0}^{\infty} I_{\nu}(d, i) d \nu
$$

which, upon substitution of (1), yields the instantaneous intensity distribution in the fringe pattern of the oscillating etalon. The actually observed time average, then, is

$$
\left\langle I\left(d_{0}, d_{1}, i\right)\right\rangle=(1 / 2 \pi) \int_{0}^{2 \pi} I\left(d_{0}+d_{1} \cos \omega t, i\right) d(\omega t) .
$$

It is probably impossible to solve this integral in a closed form. A solution can be obtained, however, by expanding the integrand into a Fourier series as follows.

In the usual derivation of the Airy formula (2), the complex amplitude of the wave transmitted by the etalon is obtained as [4]

$$
P=T \sum_{j} R^{j} e^{-i j \varphi}
$$

The intensity is

$$
\begin{aligned}
A & =P P^{*}=T^{2} \sum_{j, k} R^{j} R^{k} e^{-i j \varphi} e^{i k \varphi} \\
& =T^{2}\left(\sum_{n=0}^{\infty} R^{2 n}+\sum_{n=1}^{\infty}\left(e^{i n \varphi}+e^{-i n \varphi}\right) \sum_{m=0}^{\infty} R^{n+2 m}\right) \\
& =T^{2} /\left(1-R^{2}\right)\left(1+2 \sum_{n=1}^{\infty} R^{n} \cos n \varphi\right) .
\end{aligned}
$$

Thus,

$$
I(d, i)=C T^{2} /\left(1-R^{2}\right)\left[\int_{0}^{\infty} e^{-(\alpha / \beta)^{2}(\varphi-\phi)^{2}} d(\varphi / \beta)+2 \sum_{n=1}^{\infty} R^{n} \int_{0}^{\infty} e^{-(\alpha / \beta)^{2}(\varphi-\phi)^{2}} \cos n \varphi d(\varphi / \beta)\right] .
$$

Since $e^{-(\alpha / \beta)^{2} x^{2}}$ is essentially zero for $-\infty<x<-\phi$, we have

$$
\begin{aligned}
& \int_{0}^{\infty} e^{-(\alpha / \beta)^{2}(\varphi-\phi)^{2}} \cos n \varphi d \varphi \doteq \cos n \phi \int_{-\infty}^{+\infty} e^{-(\alpha / \beta)^{2} x^{2}} \cos n x d x \\
&-\sin n \phi \int_{-\infty}^{+\infty} e^{-(\alpha / \beta)^{2} x^{2}} \sin n x d x=\cos n \phi(\sqrt{\pi} \beta / \alpha) e^{-(n \beta / 2 \alpha)^{2}}-0 .
\end{aligned}
$$

Therefore,

$$
I(d, i)=K\left(1+2 \sum_{n=1}^{\infty} R^{n} e^{-(n \beta / 2 \alpha)^{2}} \cos n \phi\right),
$$

with

$$
K=\sqrt{\pi} / \alpha \cdot C T^{2} /\left(1-R^{2}\right) .
$$


For the oscillating etalon,

and

$$
\begin{aligned}
\phi & =\left(4 \pi \nu_{0} / c\right)\left(d_{0}+d_{1} \cos \omega t\right) \cos i \\
& =\phi_{0}+\phi_{1} \cos \omega t,
\end{aligned}
$$

$$
\beta / 2 \alpha=(2 \pi / \alpha c)\left(d_{0}+d_{1} \cos \omega t\right) \cos i .
$$

Neglecting the time as well as the angular dependence of the exponential function, we obtain

$$
I\left(d_{0}, d_{1}, i\right)=I\left(\phi_{0}, \phi_{1}\right)=K\left[1+2 \sum_{n=1}^{\infty} R^{n} e^{-\left(2 n \pi d_{0} / \alpha c\right)^{2}} \cos \left(n \phi_{0}+n \phi_{1} \cos \omega t\right)\right],
$$

where $\phi_{0}$ determines the static pattern, and $\phi_{1}$ describes the vibration amplitude. This is the Fourier expansion of the integrand in (8).

Hence,

With

$$
\left\langle I\left(\phi_{0}, \phi_{1}\right)\right\rangle=(K / 2 \pi)\left[\int_{0}^{2 \pi} d(\omega t)+2 \sum_{n=1}^{\infty} R^{n} e^{-\left(2 n \pi d_{0} / \alpha c\right)^{2}} \int_{0}^{2 \pi} \cos \left(n \phi_{0}+n \phi_{1} \cos \omega t\right) d(\omega t)\right] .
$$

$$
\begin{aligned}
\int_{0}^{2 \pi} \cos \left(n \phi_{0}+n \phi_{1} \cos \omega t\right) d(\omega t)=\cos n \phi_{0} & \int_{0}^{2 \pi} \cos \left(n \phi_{1} \cos \omega t\right) d(\omega t) \\
& -\sin n \phi_{0} \int_{0}^{2 \pi} \sin \left(n \phi_{1} \cos \omega t\right) d(\omega t)=2 \pi \cos n \phi_{0} J_{0}\left(n \phi_{1}\right)+0,
\end{aligned}
$$

where $J_{0}$ is the Bessel function of zero order, we finally obtain

$$
\left\langle I\left(\phi_{0}, \phi_{1}\right)\right\rangle=K\left[1+2 \sum_{n=1}^{\infty} R^{n} e^{-\left(2 n \pi d_{0} / \alpha c\right)^{2} \cos n \phi_{0}} J_{0}\left(n \phi_{1}\right)\right] .
$$

This is the desired time-average of the intensity distribution in the fringe pattern of the oscillating etalon.

\section{Discussion}

\subsection{Calculation of $2 \pi d_{0} / \alpha c$}

For the experiments described in this paper, the green line of $\mathrm{Hg} 198$ was used. Its half-width is, approximately, $2\left(\nu-\nu_{0}\right)_{1 / 2}=8 \cdot 10^{8} \mathrm{sec}^{-1}$. Thus, from (5),

and

$$
\begin{aligned}
e^{-\alpha^{2}\left(4 \cdot 10^{3} \sec ^{-1}\right)^{2}} & =\frac{1}{2}, \\
\alpha^{2} 16 \cdot 10^{16} \sec ^{-2} & =0.7, \\
\alpha & =2.1 \cdot 10^{-9} \text { sec, }
\end{aligned}
$$

$$
2 \pi d_{0} / \alpha c=d_{0} \cdot 10^{-1}
$$

with $d_{0}$ in $\mathrm{cm}$.

\subsection{The Long Etalon: Fringe Disappearance}

For the experiment of figure 1 , the data of the etalon were $d_{0}=10 \mathrm{~cm}$ and $R=0.8$. Thus, from (15) and (16),

$$
\left\langle I\left(\phi_{0}, \phi_{1}\right)\right\rangle=K\left[1+2 \sum_{n=1}^{\infty}(0.8)^{n} e^{-n^{2}} \cos n \phi_{0} J_{0}\left(n \phi_{1}\right)\right] .
$$

This series converges very fast. To obtain $\left\langle I\left(\phi_{0}, \phi_{1}\right)\right\rangle /\langle I(0,0)\rangle$ to better than four decimals, it suffices to consider the first two terms of the sum only; $n \leq 2$. 
A plot of $\langle I\rangle$ versus $\phi_{1}$ is shown in figure 3 for $\phi_{0}=2 m \pi$ (bright stationary fringes), $\phi_{0}=(2 m+1) \pi$ (dark stationary fringes), and $\phi_{0}=\left(2 m+\frac{1}{2}\right) \pi ; m=0, \pm 1, \pm 2, \ldots$ The first two curves show maxima, or minima, at $\phi_{1}=0,3.9,7.1, \ldots$ For intermediate amplitudes, $\phi_{1}=2.4,5.5, \ldots$, the three curves practically cross each other in one point.

The intensity distribution in the fringe pattern $\left(\langle I\rangle\right.$ versus $\left.\phi_{0}\right)$ is shown in figure 4 for these significant values of $\phi_{0}$. The pattern practically disappears for $\phi_{1}=2.4$ and 5.5. It reappears as a "negative" (dark fringes instead of bright ones) for $\phi_{1}=3.9$, and as a "positive" (fringes in their original positions) for $\phi_{1}=7.1$. As $\phi_{1}$ increases, the contrast decreases.

The peak voltages $V_{1}$ at which these conditions were actually observed are listed in table 1 ; the corresponding vibration amplitudes $d_{1}$ are proportional to these voltages. As must be expected from (12) if the theory is correct, the ratio $\phi_{1} / V_{1}$ is a constant, here equal to $0.035 \mathrm{v}^{-1}$.

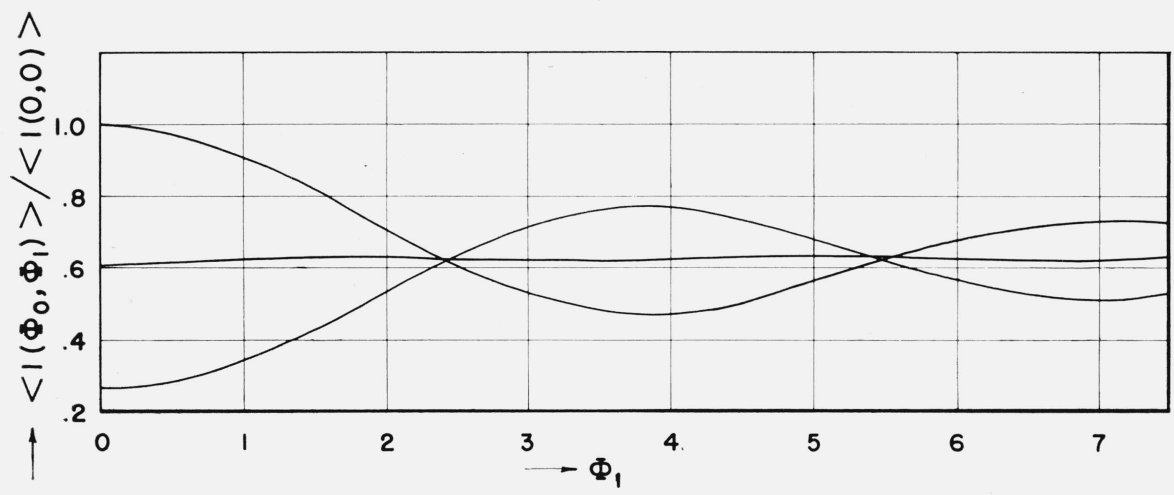

Figure 3. < I versus $\phi_{1}$ for $\phi_{0}=2 \mathrm{~m} \pi,(2 \mathrm{~m}+1 / 2) \pi$, and $(2 \mathrm{~m}+1) \pi$. $10=\mathrm{cm}$ etalon.

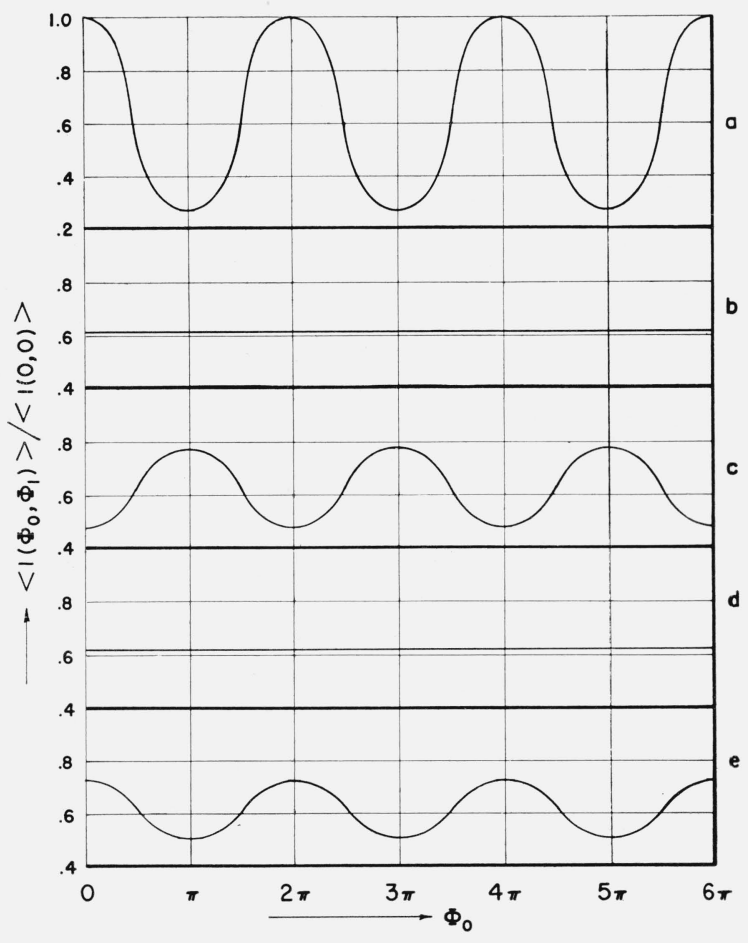

Figure 4. Theoretical intensity distribution in fringe pattern of 10-cm etalon, for $\phi_{1}=0,2.4$, $3.9,5.5$, and $\% .1$. 
TABLE 1. Significant amplitudes, $\phi_{1}$, and peak voltages, $\mathrm{V}_{\mathrm{i}}$, for $10-\mathrm{cm}$ etalon

\begin{tabular}{|c|c|c|c|}
\hline Pattern & $\phi_{1}$ & $V_{1}$ & $\phi_{1} / V_{1}$ \\
\hline $\begin{array}{l}\text { Disappeared } \\
\text { Negative } \\
\text { Disappeared } \\
\text { Positive }\end{array}$ & $\begin{array}{l}2.4 \\
3.9 \\
5.5 \\
7.1\end{array}$ & $\begin{array}{c}\text { Volts } \\
68 \\
110 \\
154 \\
201\end{array}$ & $\begin{array}{l}\text { Volt-1 } \\
0.035 \\
.036 \\
.036 \\
.035\end{array}$ \\
\hline
\end{tabular}

\subsection{The Short Etalon: Fringe Splitting}

For figure 2, an etalon of $8 \mathrm{~mm}$ length and, again, 80 percent reflectivity was used. Therefore, from (15) and (16),

$$
\left\langle I\left(\phi_{0}, \phi_{1}\right)\right\rangle=K\left[1+2 \sum_{n=1}^{\infty}(0 \cdot 8)^{n} e^{-6 n^{2} \cdot 10^{-3}} \cos n \phi_{0} J_{0}\left(n \phi_{1}\right)\right]
$$

The convergence of this expansion is rather poor. It takes 10 terms of the sum; $n \leq 10$, to obtain $\left\langle I\left(\phi_{0}, \phi_{1}\right)\right\rangle /\langle I(0,0)\rangle$ to about two decimals. This accuracy, however, is sufficient for our purpose.

The plot of $\langle I\rangle$ versus $\phi_{1}$, again for $\phi_{0}=2 m \pi,(2 m+1) \pi$, and $(2 m+1 / 2) \pi$, is shown in figure 5. Quite contrary to the corresponding graph in figure 3, the three curves cross each other in no single point. The fringe pattern can therefore no longer be expected to disappear at certain vibration amplitudes. The curves for $\phi_{0}=2 m \pi$ and $(2 m+1) \pi$ are no longer mirror images of each other; they have maxima at $\phi_{1}=0,6.4, \ldots$, and $\phi_{1}=3.4, \ldots$., respectively. The curve for $\phi_{0}=(2 m+1 / 2) \pi$, practically a straight horizontal line in figure 3 , now has pronounced peaks also; at $\phi_{1}=1.7,4.9, \ldots$

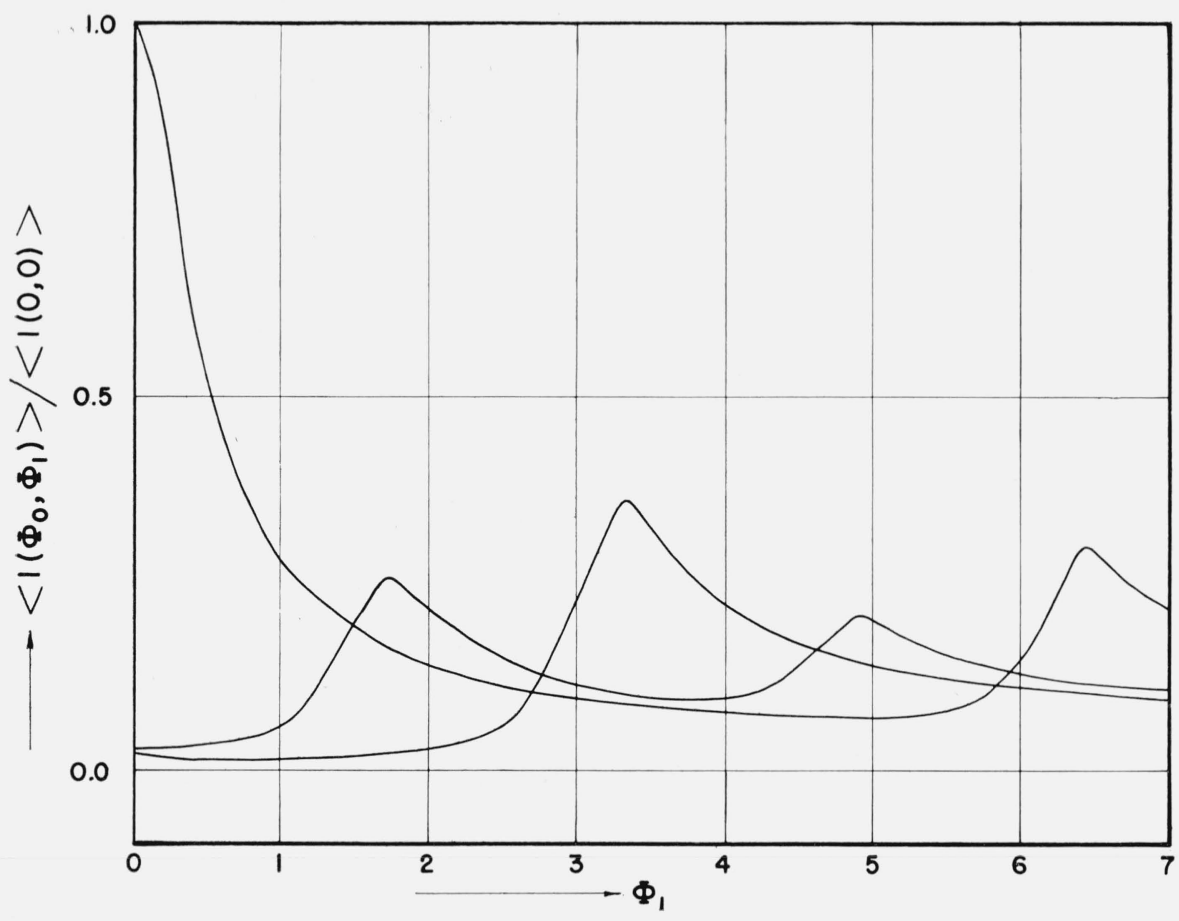

Figure 5. $<\mathrm{I}>$ versus $\phi_{1}$ for $\phi_{0}=2 \mathrm{~m} \pi,(2 \mathrm{~m}+1 / 2) \pi$, and $(2 m+1) \pi$. $8=\mathrm{mm}$ etalon. 


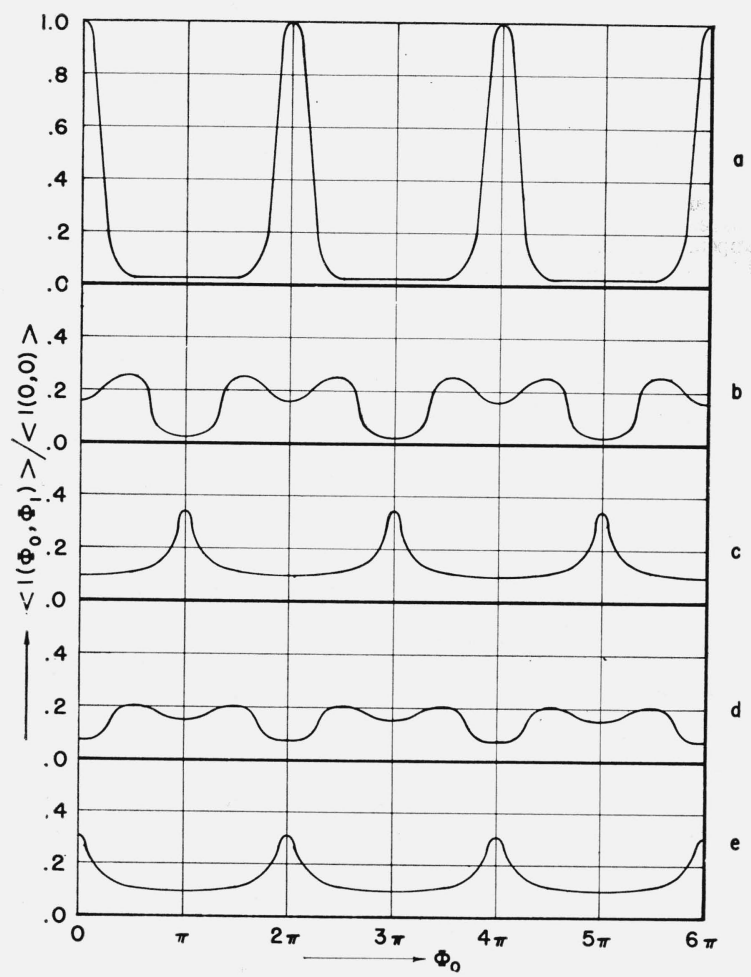

Figure 6. Theoretical intensity distribution in fringe pattern of $8-\mathrm{mm}$ etalon, for $\phi_{1}=0,1.7,3.4$, 4.9 , and 6.4 .

Figure 6 shows $\langle I\rangle$ versus $\phi_{0}$, the intensity distribution in the fringe pattern. One sees, as in figure 2, (a), the stationary pattern at $\phi_{1}=0,(\mathrm{~b})$, the split "positive" pattern at $\phi_{1}=1.7$, (c), the "negative" pattern at $\phi_{1}=3.4$, (d), the split "negative" pattern at $\phi_{1}=4.9$, and (e), the reappearance of a "positive" pattern at $\phi_{1}=6.4$. Again, the overall contrast decreases as $\phi_{1}$ increases.

The peak voltages $V_{1}$ at which the photographs in figure 2 were taken are compared to these $\phi_{1}$-values in table 2. Again, the agreement between experiment and theory is satisfactory as $\phi_{1} / V_{1}$ turns out to be the same constant as in table 1 .

\begin{tabular}{c|c|c|c} 
TABLE 2. Significant amplitudes, $\phi_{1}$, and peak voltages, $\mathrm{V}_{1}$, \\
for 8-mm etalon
\end{tabular}

\section{Conclusions}

The fringe pattern of oscillating Fabry-Perot etalons, as shown in figures 1 and 2, are fully explained by the above theory. Fringe disappearance, such as also obtained with an oscillating two-beam interferometer, is observed when the fringes in the stationary pattern are broad. Fringe splitting is found when they are narrow. The vibration amplitudes significant for fringe reappearance, and disappearance or splitting, are different in the two cases.

The behavior of the pattern, thus, depends entirely upon experimental circumstances such as, the length of the etalon, the reflectance of the etalon plates, and the width of the 
spectrum line used for illumination. Mathematically, these factors determine the rate of convergence of the series expansion (15) and, thus, the distinction between the two border cases.

For an oscillating two-beam interferometer, no such dependence upon experimental circumstances exists. For the measurement of vibration amplitudes, therefore, an oscillating multiple-beam interferometer appears to be less practicable than a two-beam interferometer.

\section{References}

[1] K. D. Mielenz, R. B. Stephens, and K. F. Nefflen, J. Res. NBS $68 C$ (Eng. and Instr.) No. 1, 1-6 (1964).

[2] H. Osterberg, J. Opt. Soc. Am. 22, 19 (1932).

[3] For instance: V. A. Schmidt, S. Edelman, E. R. Smith, and E. T. Pierce, J. Acoust. Soc. Am. 34, 455 (1962).

[4] R. W. Ditchburn, Light, p. 138 (Interscience Publishers Inc., New York 1961).

(Paper 68C2-152) 